\title{
Fetal Alcohol Spectrum Disorder
}

National Cancer Institute

\section{Source}

National Cancer Institute. Fetal Alcohol Spectrum Disorder. NCI Thesaurus. Code C92780.

A group of disorders caused by a prenatal exposure to maternal consumption of alcohol leading to a range of behavioral, cognitive and neurological deficits in the offspring. It is characterized by physical growth problems, distinct facies, and varying psychoneurological issues. 